\title{
Relative Complexity Index for Decision-Making Method
}

\author{
Harliza Mohd Hanif*, Daud Mohamad, Rosma Mohd Dom \\ Faculty of Computer \& Mathematical Sciences, University Teknologi MARA, 40450 Shah Alam, Selangor Darul Ehsan, Malaysia
}

Received February 1, 2021; Revised April 2, 2021; Accepted April 18, 2021

\begin{abstract}
Cite This Paper in the following Citation Styles
(a): [1] Harliza Mohd Hanif, Daud Mohamad, Rosma Mohd Dom, "Relative Complexity Index for Decision-Making Method," Mathematics and Statistics, Vol. 9, No. 3, pp. 233 - 242, 2021. DOI: 10.13189/ms.2021.090304.
\end{abstract}

(b): Harliza Mohd Hanif, Daud Mohamad, Rosma Mohd Dom (2021). Relative Complexity Index for Decision-Making Method. Mathematics and Statistics, 9(3), 233 - 242. DOI: 10.13189/ms.2021.090304.

Copyright $(2021$ by authors, all rights reserved. Authors agree that this article remains permanently open access under the terms of the Creative Commons Attribution License 4.0 International License

\begin{abstract}
The complexity of a method has been discussed in the decision-making area since complexity may impose some disadvantages such as loss of information and a high degree of uncertainty. However, there is no empirical justification to determine the complexity level of a method. This paper focuses on introducing a method of measuring the complexity of the decision-making method. In the computational area, there is an established method of measuring complexity named Big-O Notation. This paper adopts the method for determining the complexity level of the decision-making method. However, there is a lack of applying Big-O in the decision-making method. Applying Big-O in decision-making may not be able to differentiate the complexity level of two different decision-making methods Hence, this paper introduces a Relative Complexity Index (RCI) to cater to this problem. The basic properties of the Relative Complexity Index are also discussed. After the introduction of the Relative Complexity Index, the method is implemented in Technique for Order of Preference by Similarity to Ideal Solution (TOPSIS) method.
\end{abstract}

Keywords Complexity, Relative Complexity Index, Big-O Notation

\section{Introduction}

The complexity of a method is having many parts that relate to one another. The level of complexity of a method is different from one another. Many areas already acknowledge the importance of determining the complexity level of a method since having high complexity level may impose several disadvantages. For example, in decision-making method discussed that complexity in a method imposes a high degree of uncertainty and unpredictability regarding the outcomes of managerial decision-making for example in terms of accuracy [1] and timing [2]. Supported by [3], complexity reduces the awareness of decision-makers and thus the efficiency of their decisions. As mentioned by [4] also, complexity may impose a high computational cost on the operation.

In the decision-making method, [5]-[7] have addressed the complexity issue in the decision-making method and it always a claim that one method is better and simple as compared to others. However, this claim lacks empirical justification. Thus, there is a need to measure the complexity level of each decision-making method. Furthermore, the importance of determining the complexity level is also supported by [8], [9]. Hence, this paper proposed a method to determine the complexity level of the decision-making method. The latter section is the introduction of complexity. The sub-section of complexity is Big-O in computational, the sample of calculation based on Big-O, Relative Complexity Index for decision-making, the general form of complexity index based on Big-O and RCI, and Properties of the RCI. The implementation of the proposed method is in the next section. The conclusion of this paper is in the final section.

\section{Complexity}

Making good decisions are very essential in any organization. However, the nature of the problems to be solved is becoming more complicated as opposed to definiteness; elements of subjectivity and vagueness are now common. Thus, decision-making methods incorporated the decision-makers' opinions in determining 
the solution. Decision-making problems can be divided into two parts; that is homogeneous and heterogeneous. Based on Kanazawa (1998), heterogeneous group decision making means the opinions of individuals are allowed, which is contrary to the homogeneous group decision-making environment. Jiang, Fan, \& Ma (2008) claimed that it is difficult to agree on the number of linguistic terms sets to be used by the decision-maker in evaluating the alternative due to the differences in cultural, educational backgrounds, experience, and knowledge of the decision-makers. This, by all means, homogeneous group decision-making demonstrated that all decision makers must have common agreement on linguistic term sets and from the same type of scale. Meanwhile, heterogeneous group decision making authorized the linguistic term sets to be varied (multi-granular linguistic term sets) or in a different type of scale. The illustration of the decision-making problems, in general, is as Figure 1.

Meanwhile, the illustrations of the homogeneous group decision-making process and heterogeneous group decision-making process are presented in Figure 2 and Figure 3 respectively.

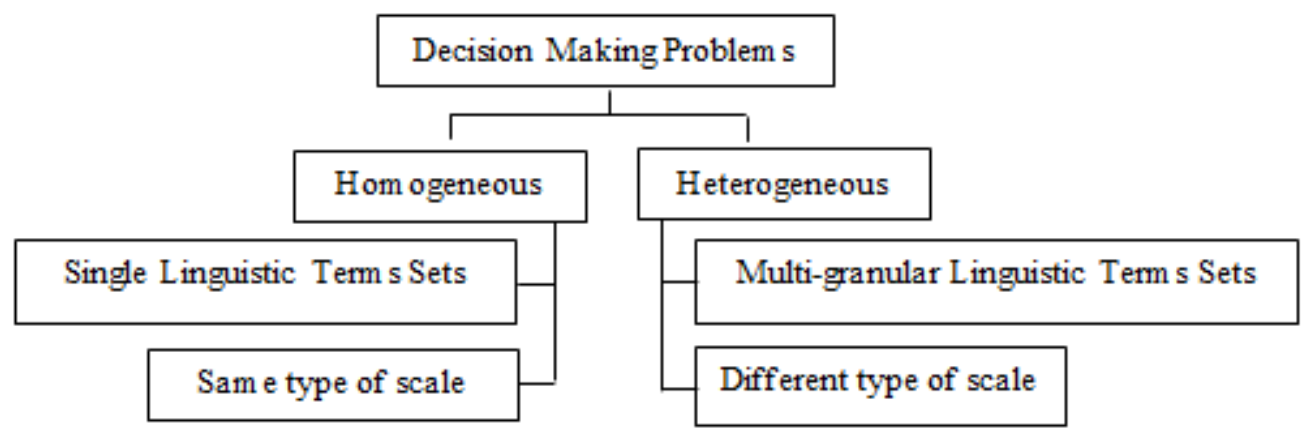

Figure 1. The illustration of decision-making problems

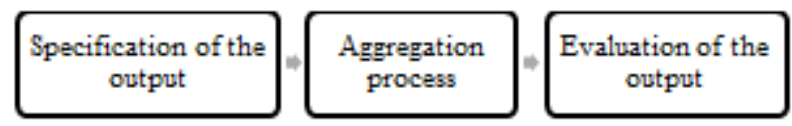

Figure 2. Homogeneous group decision-making process

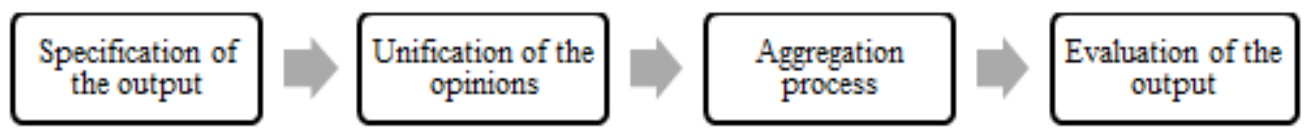

Figure 3. Heterogeneous group decision-making process

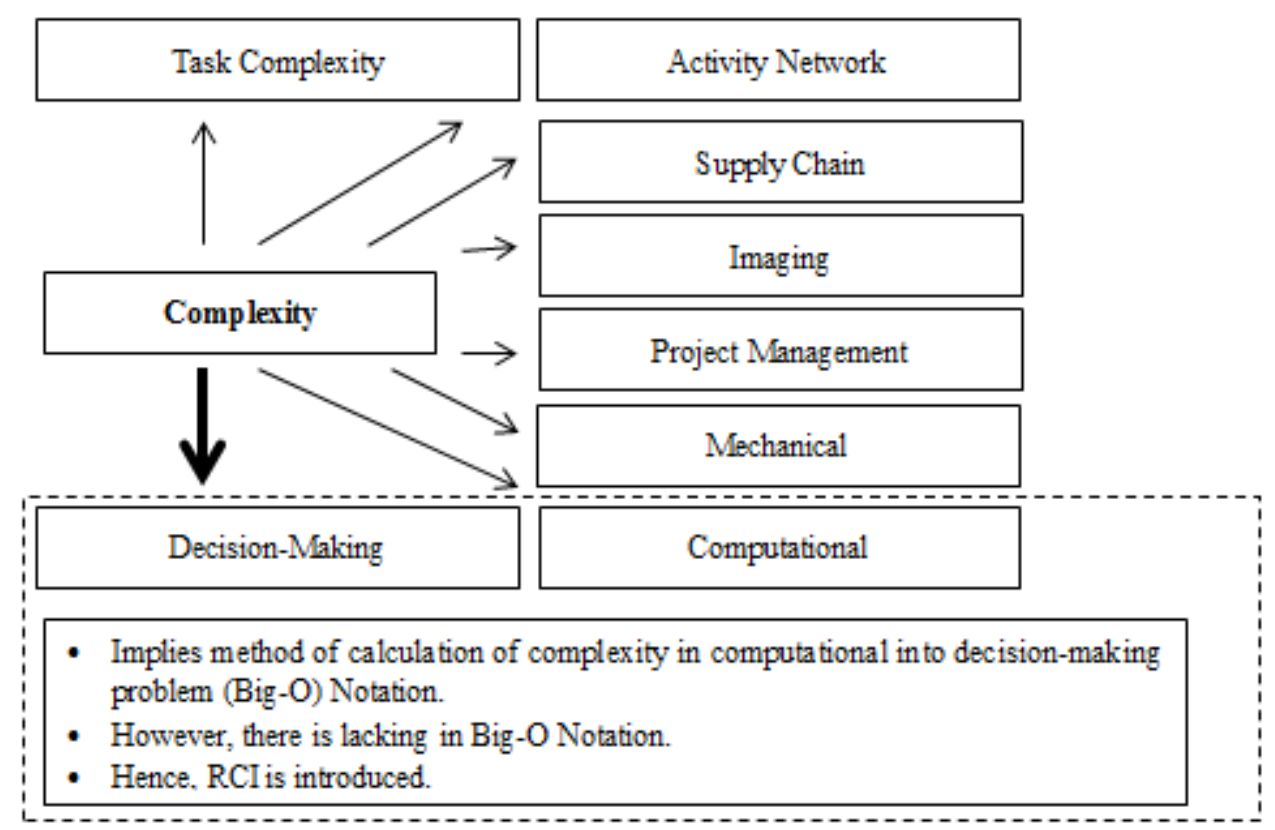

Figure 4. The overview of the research 
Since the computational field already established a well-known method of complexity (Big-O Notation), the proposed method of complexity for the decision-making problem is referred to it (computational method). The overview of the paper is as figure 4 .

\subsection{Big-O in Computational}

In the computational field, Juris Hartmanis and Richard E. Stearns already developed a measure of the difficulty degree of an algorithm to compare the efficiency of the algorithm. Based on computational complexity, there are two factors to be considered; time and space. [12] claimed that the time factor is more important than space. The efficiency of the algorithm is usually focused on the amount of time elapsed when processing data. An algorithm by [13] is defined as a step-by-step procedure for performing some tasks in a finite amount of time. According to [13], the running time of an algorithm or data structure method increases with the input data.

The Big Notation (Big-O) is frequently used to characterize running times and space bounds in terms of some parameter $n$, which varies from one problem to problem, but it is always defined as a chosen measure of the "size" of the problem in computational complexity. The Big-O notation is introduced by Paul Bachmann in 1894. The main objective of Big-O is to determine the general dependence of running time on the size of the input. It is then visualized by plotting the performance of each run of the algorithm as a point with $x$-coordinate equal to the input size, $n$, and $y$-coordinate equal to the running time, $t$. The graph is shown in figure 5 .

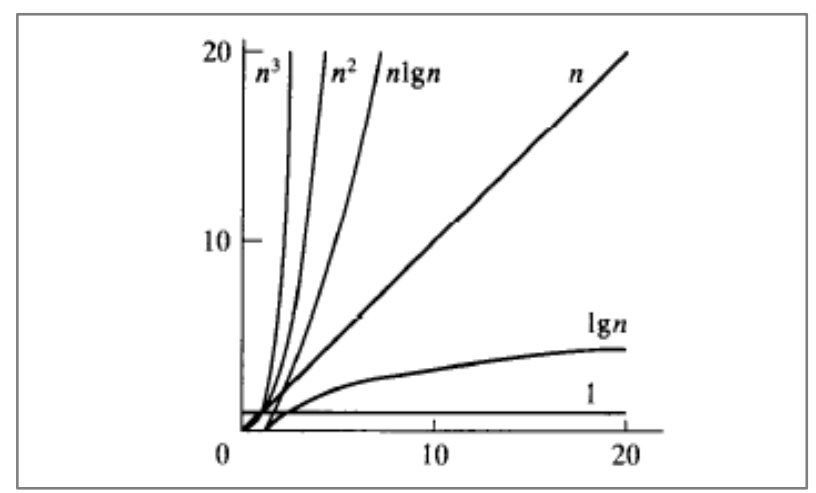

Figure 5. Types of functions applied in Big-O notation

Based on [13], there are seven functions commonly used in conjunction with the Big-O notation to characterize the running times and space usage of the algorithm [13]. Based on [13], the common functions are; constant, logarithm, linear, $\mathrm{N}-\log -\mathrm{N}$, quadratic, cubic function, and other polynomials and exponential functions. The definition of growth rate is the change of processer running time within the specific input size. The growth rate of the functions is as Table 1.

Table 1. The growth rate of the functions

\begin{tabular}{|c|c|c|c|c|}
\hline No & & Function & Big-O Notation & Note \\
\hline 1 & $\begin{array}{l}\text { i. } \\
\text { ii. } \\
\text { a) } \\
\text { b) } \\
\text { c) } \\
\text { d) } \\
\text { e) } \\
\text { f) } \\
\text { g) }\end{array}$ & $\begin{array}{l}\text { Constant } \\
\text { Primitive Operations } \\
\text { Assigning a value to a variable } \\
\text { Calling a function } \\
\text { Performing an arithmetic operation (for } \\
\text { example, adding two numbers) } \\
\text { Comparing two numbers } \\
\text { Indexing into an array } \\
\text { Following an object reference } \\
\text { Returning from a function }\end{array}$ & $\mathrm{O}(1)$ & Consisting of basic operations. \\
\hline 2 & & Logarithm & $\mathrm{O}(\log n)$ & The larger the base, the more complex the logarithm. \\
\hline 3 & & Linear & $\mathrm{O}(n)$ & \multirow{2}{*}{ The higher the value of $n$, the higher the complexity. } \\
\hline 4 & & Linearithmic, log-linear, quasilinear & $\mathrm{O}(n \log n)$ & \\
\hline 5 & & $\begin{array}{l}\text { uadratic, Fractional power, polynomial or } \\
\text { algebraic }\end{array}$ & $\mathrm{O}\left(n^{c}\right)$ & $\begin{array}{l}\text { The complexity of polynomials increases as the } \\
\text { exponent increases }\end{array}$ \\
\hline 6 & & Exponential & $\mathrm{O}\left(c^{n}\right)$ & If the base is the same, complexity increases with power. \\
\hline 7 & & Factorial & $\mathrm{O}(n !)$ & The higher the value of $n$, the higher the complexity. \\
\hline
\end{tabular}




\subsection{Sample of Calculation Based on Big-O Notation}

To make samples of calculation of function based on Big-O, assume there are four functions to be evaluated in terms of their efficiency. The four functions are as follows;

Table 2. Sample of complexity calculation based on Big-O notation

\begin{tabular}{cl}
\hline Function & Big-O \\
\hline$f_{1}(x)=4 x^{2}+x-1$ & $O\left(x^{2}\right)$ \\
\hline$f_{2}(x)=4 x^{2}$ & $O\left(x^{2}\right)$ \\
\hline$f_{3}(x)=\log x+7 x^{4}$ & $O\left(x^{4}\right)$ \\
\hline$f_{4}(x)=\frac{1}{2} x+5 x !$ & $O(x !)$ \\
\hline
\end{tabular}

Referring to Table 2, based on Big-O notation, function $f_{4}(x)$ has the highest complexity level. The second highest is the function $f_{3}(x)$. The least complex function is $f_{1}(x)$ and $f_{2}(x)$. Big-O notation ranks two functions as having the same level of complexity despite the difference of elements of the function. Function $f_{1}(x)$ has more steps compare to function $f_{2}(x)$. This is due to Big-O notation allows disregarded constant factors and lower order terms and focusing on the main components of a function that affect its growth. Thus, this may allow for several decision-making methods of having the same complexity level when it is not. The extension of Big-O notation is essential to differentiate the complexity of each decision-making method. If exist more than one decision-making method with the same conclusion based on Big-O notation, it is required to proceed to the Relative Complexity Index (RCI) to determine the complexity of the method.

\subsection{Relative Complexity Index for Decision-Making}

[13] in their study revealed that it is possible to analyze a particular algorithm without performing experiments on its running time. Commonly, it is related to the primitive operation which corresponds to a low-level instruction with an execution time that is constant. Hence, instead of trying to determine the specific execution time of each primitive operation, [13] simply count how many primitive operations are executed. According to them, this operation count correlates to actual running time in a specific computer, since each primitive operation corresponds to a constant-time instruction, and there are only a fixed number of primitive operations. Also, the assumption in this approach is that the running times of different primitive operations are fairly similar. As a result, the number of primitive operations of an algorithm performs is proportional to the actual running time of that algorithm. The definition of primitive operations such as the following:

i. Assigning a value to a variable

ii. Calling a function

iii. Performing an arithmetic operation (for example, adding two numbers) iv. Comparing two numbers

v. Indexing into an array

vi. Following an object reference

vii. Returning from a function

Based on [13], method on how to determine the complexity of the primitive operation, this research proposed the Complexity Index (CI) and The Relative Complexity Index (RCI). To begin with, the CI and RCI for the decision-making method are defined.

Let $p$ be the representation of discrete sets of the method to be evaluated such that, $\left\{\operatorname{Method}_{1}, \operatorname{Method}_{2}, \operatorname{Method}_{3}, \ldots, \operatorname{Method}_{p}\right\}$ and $S T_{q}$ be the step for each method such that $\left\{S T_{1}, S T_{2}, S T_{3}, \ldots, S T_{q}\right\}$

Definition 1 Complexity Index (CI) of $\operatorname{Method}_{p}$ is defined as the summation of primitive operations in a decision-making method for each step.

$$
\sum_{S T_{1}}^{S T_{q}} C I_{\text {Method }_{p}}
$$

Definition 2 Relative Complexity Index (RCI) of Method $_{p}$ is defined as the normalized value of the Complexity Index (CI) of a decision-making method. The normalized process will result in the value of $\mathrm{RCI}$ to be in the range of $(0,1]$.

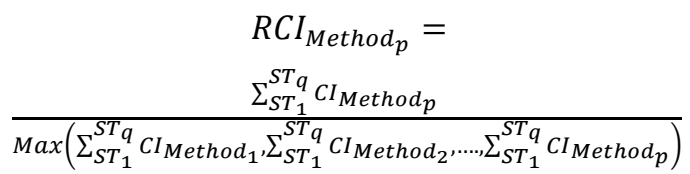

The domain of the RCI, Dom (RCI) is any natural number and the range, Range (RCI) is between zero and one $(0,1]$.

$$
\begin{aligned}
\text { Dom }(\mathrm{RCI}) & =\{N\} \\
\text { Range }(\mathrm{RCI}) & =(0,1]
\end{aligned}
$$

The measurement of the complexity index is considered the worst-case scenario as [13] claimed it would be more realistic in terms of identifying the complexity level of the case. For example, if in a decision-making method exists a step with two sub-steps to be chosen, chose the sub-step with a higher $\mathrm{CI}$ in determining the complexity index.

The examples of obtaining the CI of mathematical formulas in unification, aggregation, and evaluation process are as follows;

$$
\text { 1. } x=\frac{m}{\sqrt{a+b}}, \mathrm{CI}=3
$$

There are three types of operations involved which are addition, square root, and division. The first operation is ' $a+b$ '. Then $\sqrt{a+b}$, is considered as one operation. Follows by the division of $m$ and ' $\sqrt{a+b}$ ' that will require one more operation. Thus, the total number of operations involves for this function is three.

2. $y=\left(\sum_{j=1}^{3} a-b\right), \mathrm{CI}=5$

There are two types of operations involved which are subtraction and summation. The first operation is requiring to find $a-b$. Since it involves three values, the total 
operation is three $(1 \times 3=3)$. Subsequently, the summations of the three values $(j=1,2,3)$ are two additional operations. Thus, the total number of operations for this function is five.

\subsection{The General Form of Complexity Index Based on Big-O and RCI}

The proposed procedure method of ranking the method based on the complexity level is presented as follows;

\begin{tabular}{|c|c|c|}
\hline Step 1: & \multicolumn{2}{|c|}{$\begin{array}{l}\text { Determine the methods of } \\
\text { decision-making method to be evaluated } \\
\left\{\operatorname{Method}_{1}, \operatorname{Method}_{2}, \operatorname{Method}_{3}, \ldots, \text { Method }_{p}\right\}\end{array}$} \\
\hline Step 2: & \multicolumn{2}{|c|}{$\begin{array}{l}\text { Determine the complexity of each of the } \\
\text { selected methods based on Big-O. }\end{array}$} \\
\hline Step 3: & $\begin{array}{l}\text { Rank t } \\
\text { level a } \\
\text { on the }\end{array}$ & $\begin{array}{l}\text { method based on the complexity } \\
\text { cordingly. The ranking will depend } \\
\text { ollowing two cases; } \\
\text { The complexity of all selected } \\
\text { methods managed to be } \\
\text { evaluated accordingly based on } \\
\text { Big-O. } \\
\text { Exist more than one method } \\
\text { with the same complexity level } \\
\text { based on Big-O. Hence, } \\
\text { proceed to RCI such as the } \\
\text { sub-step; }\end{array}$ \\
\hline $\begin{array}{l}\text { Sub-Step } \\
\text { 1: }\end{array}$ & \multicolumn{2}{|c|}{$\begin{array}{l}\text { decision-making with the same level of } \\
\text { complexity to be evaluated } \\
\left\{\operatorname{Method}_{1}, \operatorname{Method}_{2}, \operatorname{Method}_{3}, \ldots, \text { Method }_{p}\right\}\end{array}$} \\
\hline $\begin{array}{l}\text { Sub-Step } \\
\text { 2: }\end{array}$ & \multicolumn{2}{|c|}{$\begin{array}{l}\text { Calculate the complexity index, CI for } \\
\text { each of the selected method based on } \\
\text { equation (1). }\end{array}$} \\
\hline $\begin{array}{l}\text { Sub-Step } \\
\text { 3: }\end{array}$ & \multirow{2}{*}{\multicolumn{2}{|c|}{$\begin{array}{l}\text { Determine the maximum value of CI } \\
\text { among the selected methods } \\
\text { Evaluate the value of RCI for each of the } \\
\text { selected method based on equation (2) }\end{array}$}} \\
\hline $\begin{array}{l}\text { Sub-Step } \\
\text { 4: }\end{array}$ & & \\
\hline
\end{tabular}

\subsection{Basic Properties of RCI}

The value of RCI is in the interval $(0,1]$. To determine the properties of the RCI, Let assume there exist three decision-making methods (DMM), $\left\{D M M_{x}, D M M_{y}, D M M_{z}\right\} \quad$ with RCI such as $\left\{R C I_{x}, R C I_{y}, R C I_{z}\right\}$ accordingly.

Property 1. It is an ordered set since if $\mathrm{RCI}_{x}<\mathrm{RCI}_{y}$ and $\mathrm{RCI}_{y}<\mathrm{RCI}_{z}$, then $\mathrm{RCI}_{x}<\mathrm{RCI}_{z}$.

If there exist two or more decision-making methods integrated, the RCI has the following properties:

Property 2. Commutatively of addition $\mathrm{RCI}_{x}+\mathrm{RCI}_{y}=$ $\mathrm{RCI}_{y}+\mathrm{RCI}_{x}$ for all $x, y \in N$.

For example; if exist two different decision-making method to be combined (the process) such as $D M M_{x}+$ $D M M_{y}$, hence, the total RCI can be calculated such as $\mathrm{RCI}_{x}+\mathrm{RCI}_{y}$ or $\mathrm{RCI}_{y}+\mathrm{RCI}_{x}$.

Property 3. Associativity of addition $\left(\mathrm{RCI}_{x}+\mathrm{RCI}_{y}\right)+$ $\mathrm{RCI}_{z}=\mathrm{RCI}_{x}+\left(\mathrm{RCI}_{y}+\mathrm{RCI}_{z}\right)$ for all $x, y, z \in N$.

For example; if there exist three different decision-making methods to be combined (the process) such as $D M M_{x}+D M M_{y}+D M M_{z}$, hence, the total RCI can be calculated such as $\left(\mathrm{RCI}_{x}+\mathrm{RCI}_{y}\right)+\mathrm{RCI}_{z}$ or $\mathrm{RCI}_{x}+\left(\mathrm{RCI}_{y}+\mathrm{RCI}_{z}\right)$.

\section{The Implementation}

For implementation purposes of the RCI, the complexity level of TOPSIS for crisp value is selected. TOPSIS is a ranking and selection technique of a number externally determined by alternatives through distance measure [14]. The comparison of the complexity level is evaluated based on one and three decision-makers.

As a sample of calculation, [15] method of crisp value is selected. To determine the complexity level based on Big-O, Table 1 is referred to. Table 3 shows the dominant notation for each step that consists of function(s) for one decision-maker. 
Table 3. Sample calculation of Big-O for the crisp value of one decision-maker

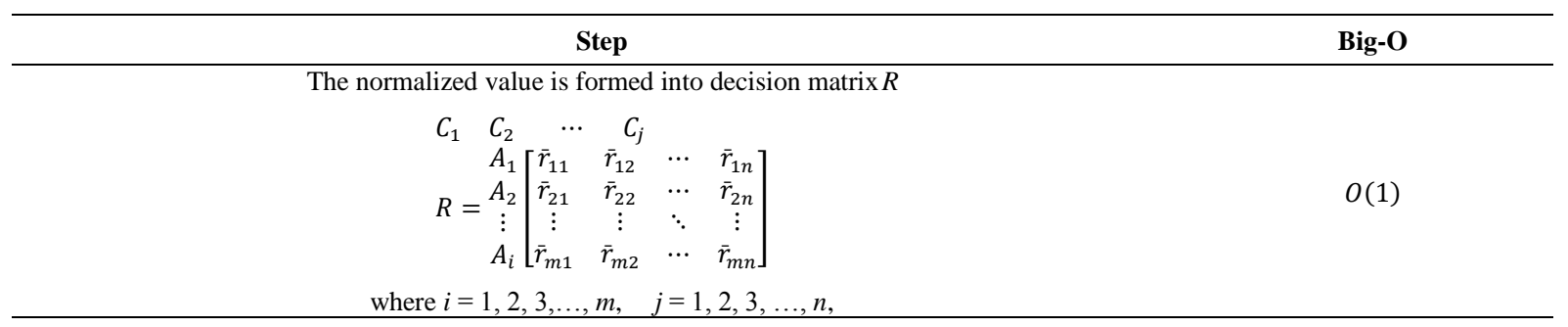

The aggregated weighted value obtained is multiplied into the normalized decision matrix to form the weighted normalized decision matrix

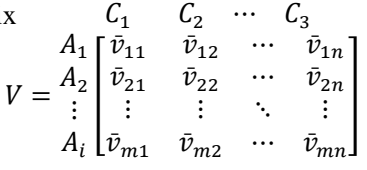

where $i=1,2,3, \ldots, m, \quad j=1,2,3, \ldots, n$, where $\bar{v}_{i j}=\bar{r}_{i j}(.) \bar{w} j$

According to the TOPSIS algorithm, PIS, $A^{+}$and NIS, $A^{-}$are identified from the weighted normalized decision matrix according based on;

$$
\begin{gathered}
A^{+}=\left\{\bar{v}_{1}^{+}, \bar{v}_{2}^{+}, \cdots, \bar{v}_{n}^{+}\right\} \text {where } \bar{v}_{j}^{+}=\max \left\{\bar{v}_{i j}\right\} \\
\text { where } i=1,2,3, \ldots, m, \quad j=1,2,3, \ldots, n, \\
A^{-}=\left\{\bar{v}_{1}^{-}, \bar{v}_{2}^{-}, \cdots, \bar{v}_{n}^{-}\right\} \text {where } \bar{v}_{j}^{+}=\min \left\{\bar{v}_{i j}\right\}
\end{gathered}
$$

The separation distance of each maintenance strategy from the PIS and NIS is calculated by using the Euclidean distance as presented;

$$
\begin{aligned}
& d_{i}^{+}=\sum_{j=1}^{n} \sqrt{\left(\bar{v}_{i j}-\bar{v}_{j}^{+}\right)^{2}}, j=1,2, \ldots, n, \\
& d_{i}^{-}=\sum_{j=1}^{n} \sqrt{\left(\bar{v}_{i j}-\bar{v}_{j}^{-}\right)^{2}}, j=1,2, \ldots, n,
\end{aligned}
$$

where $d_{i}^{+}$represents the separation of a distance of maintenance strategy $A_{i}$ from the PIS and $d_{i}^{-}$ represents the separation distance from NIS

The measurement of the closeness coefficient is conducted by referring to;

$$
C_{i}^{*}=\frac{d_{i}^{-}}{d_{i}^{+}+d_{i}^{-}}, 0<C_{i}^{*}<1, i=1,2, \ldots n .
$$

The ranking of the output based on $C_{i}^{*}$

$(O(1)$ is selected since each step only involves basic primitive operations)

Since the Big-O consider the dominant term, hence, the determinacy of the complexity level is as follows;

$$
\begin{aligned}
\text { Big-O }_{\text {TOPSIS,Crisp Value, } n(D M)=1}=\max & \left(O(1), O(1), O(1), O\left(n^{2}\right), O\left(n^{\frac{1}{2}}\right), O(1), O(1)\right) \\
& =O\left(n^{2}\right)
\end{aligned}
$$


Table 4. Sample calculation of Big-O for the crisp value of three decision-makers

\begin{tabular}{|c|c|}
\hline Step & Big-O \\
\hline $\begin{array}{l}\bar{x}_{i j}=\sum_{k=1}^{K} \frac{x_{i j k}}{K} \text { in which } x_{i j k} \text { represents the rating value of the } i \text { th maintenance strategy with the } \\
\text { respective } j \text { th factor given by decision-maker } k \text { where } i=1,2,3, \ldots, m, j=1,2,3, \ldots, n \text {, and } k=1,2, \\
3, \ldots, K\end{array}$ & $O(1)$ \\
\hline $\begin{array}{l}\bar{w}_{i j}=\sum_{k=1}^{K} \frac{w_{i j k}}{K} \text { where } \bar{w}_{i} \text { represents the average value of the weight rating corresponding to the } i \text { th } \\
\text { factor from } K \text { decision-makers. }\end{array}$ & $O(1)$ \\
\hline 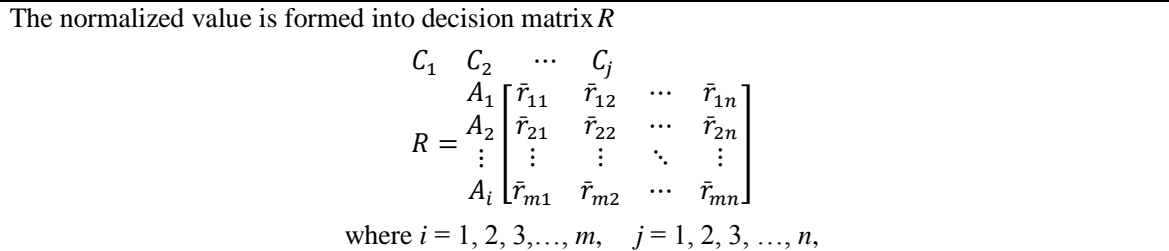 & $O(1)$ \\
\hline 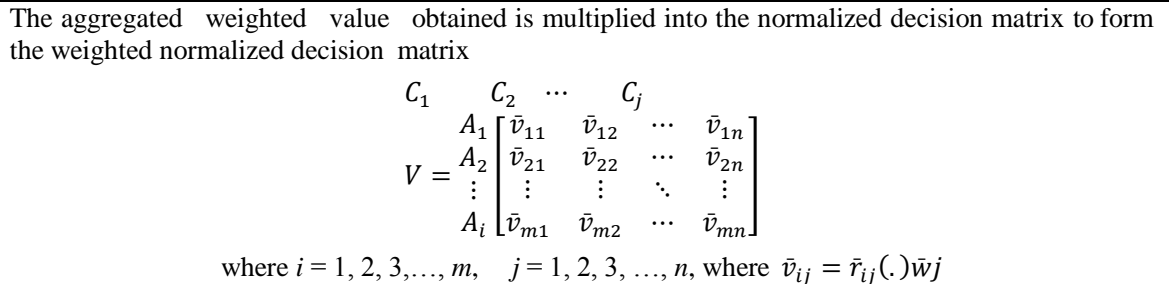 & $O(1)$ \\
\hline $\begin{array}{l}\text { According to the TOPSIS algorithm, PIS, } A^{+} \text {and NIS, } A^{-} \text {are identified from the weighted normalized } \\
\text { decision matrix according based on; } \\
\qquad \begin{array}{c}A^{+}=\left\{\bar{v}_{1}^{+}, \bar{v}_{2}^{+}, \cdots, \bar{v}_{n}^{+}\right\} \text {where } \bar{v}_{j}^{+}=\max \left\{\bar{v}_{i j}\right\} \\
\text { where } i=1,2,3, \ldots, m, \quad j=1,2,3, \ldots, n \\
A^{-}=\left\{\bar{v}_{1}^{-}, \bar{v}_{2}^{-}, \cdots, \bar{v}_{n}^{-}\right\} \text {where } \bar{v}_{j}^{+}=\min \left\{\bar{v}_{i j}\right\}\end{array}\end{array}$ & $O(1)$ \\
\hline $\begin{array}{l}\text { The separation distance of each maintenance strategy from the PIS and NIS is calculated by using the } \\
\text { Euclidean distance as presented; } \\
\qquad d_{i}^{+}=\sum_{j=1}^{n} \sqrt{\left(\bar{v}_{i j}-\bar{v}_{j}^{+}\right)^{2}}, j=1,2, \ldots, n, \\
\qquad d_{i}^{-}=\sum_{j=1}^{n} \sqrt{\left(\bar{v}_{i j}-\bar{v}_{j}^{-}\right)^{2}}, j=1,2, \ldots, n, \\
\text { where } d_{i}^{+} \text {represents the separation of a distance of maintenance strategy } A_{i} \text { from the PIS and } d_{i}^{-} \\
\text {represents the separation distance from NIS }\end{array}$ & $O\left(n^{2}\right)$ \\
\hline $\begin{array}{l}\text { The measurement of the closeness coefficient is conducted by referring to; } \\
\qquad C_{i}^{*}=\frac{d_{i}^{-}}{d_{i}^{+}+d_{i}^{-}}, 0<C_{i}^{*}<1, i=1,2, \ldots n\end{array}$ & $O(1)$ \\
\hline The ranking of the output based on $C_{i}^{*}$ & $O(1)$ \\
\hline
\end{tabular}

As a comparison, the complexity level for the same method of [15] for crisp value using Big-O is now determined. Table 4 shows the sample calculation of Big-O for the crisp value of three decision-makers.

$(O(1)$ is selected since each step only involves basic primitive operations)

Since the Big-O consider the dominant term, hence, the determinacy of the complexity level is as follows;

$$
\begin{gathered}
\text { Big-O }_{\text {TOPSIS,Crisp Value }, n(D M)=3}= \\
\max \left(O(1), O(1), O(1), O(1), O(1), O\left(n^{2}\right), O\left(n^{\frac{1}{2}}\right), O(1), O(1)\right) \\
=O\left(n^{2}\right)
\end{gathered}
$$

Based on this comparison, it shows that based on Big-O, the decision-making method of [15] for crisp value has the same complexity level for one and three decision-makers. Thus, this is under Case 2 based on the proposed method of determinacy of complexity level (Section 2.4) where more than one method with the same complexity level exists. Hence, proceed to obtain the RCI. The evaluation of CI is needed in determining the RCI of each method. Thus, forms the general form of CI of [15] decision-making method. First, let;

$n(D M)$ represents the number of decision-makers,

$n(A T)$ representing the number of alternatives,

$n(C R)$ represents the number of criteria, and

$n(P O)$ represents the value of the primitive operation.

The general form of CI for a method is formulated by determining the general form for each step first in the form of $\{n(D M), n(A T), n(C R), n(P O)\}$. Once the general form of $\mathrm{CI}$ is obtained for each step, the formula is simplified into one function for analysis purposes. The procedure of formulating the general form is as follows; 
Procedure 1: $\quad$ Selecting a method, i.e $\left\{\operatorname{Method}_{\text {TOPSIS, Crisp Value }}\right\}$

Procedure 2: The general form in terms of $\{n(D M), n(A T), n(C R), n(P O)\}$ for each step such that $\left\{S T_{1}, S T_{2}, S T_{3}, \ldots, S T_{q}\right\}$ is determined. As the sample on how the first step of Table 4 is obtained, first, let;

$$
\begin{gathered}
n(A T) \text { be the number of alternatives, } \\
n(C R) \in\left\{C R_{1}, C R_{2}, C R_{3}\right\}, \text { and } \\
n(D M) \in\left\{D M_{1}, D M_{2}, D M_{3}\right\} .
\end{gathered}
$$

Each DM will evaluate each of the AT based on the CR listed. Step 1 of the proposed method aggregates the evaluation using the average method. Hence, the general form of CI of the first step is formulated as follows.

For each alternative (AT);

$$
\begin{aligned}
\mathrm{CR}_{1} & =\frac{\mathrm{DM}_{1}+\mathrm{DM}_{2}+\mathrm{DM}_{3}}{3} \\
\mathrm{CR}_{2} & =\frac{\mathrm{DM}_{1}+\mathrm{DM}_{2}+\mathrm{DM}_{3}}{3} \\
\mathrm{CR}_{3} & =\frac{\mathrm{DM}_{1}+\mathrm{DM}_{2}+\mathrm{DM}_{3}}{3}
\end{aligned}
$$

The general form of CI for summation; $(n(D M)-1)$

Step 1 Furthermore, for the general form of CI for division; 1

Hence, the general form is;

$$
(n(D M)-1)+1=n(D M)
$$

Since the number of DM depends on the number of CR, the general form of $\mathrm{CI}$ is

Step 2 follow;

$$
n(D M) \times n(C R)
$$

The general form of $\mathrm{CI}$ is also depending on the number of AT. Hence, the final gen Step 3 form of CI is as follow;

$$
n(D M) \times n(C R) \times n(A T)
$$

Procedure 3: After the general form of $\mathrm{CI}$ is formed (Table 4) for each step, each of the general forms of CI will be combined and simplified. The general expression for the CI can be divided into two cases that are when $\square \square \square=1$ and $\square \square \square>1$.

\section{Case 1}

$$
\begin{gathered}
\text { When }(n(D M)=1) \\
C I_{\text {TOPSIS,Crisp Value }, n(D M)=1}=(2+2 \times n(A T) \times n(C R))+(n(A T) \times n(C R))+ \\
\begin{array}{cc}
(2+2 \times n(A T))+2 \times( & (3 \times n(A T)+n(C R)-1)+(2 \times n(A T)) \\
+1 \quad & 3+(3 \times n(A T) \times n(C R))+(10 \times n(A T))+ \\
(2 \times n(C R))
\end{array}
\end{gathered}
$$

Case 2

$$
\begin{aligned}
& \text { When }(n(D M)>1) \\
& \text { CI }_{\text {TOPSIS,Crisp Value, } n(D M)>1}=(2 \times n(D M) \times n(A T) \times n(C R))+ \\
& (2+2 \times n(A T) \times n(C R))+(n(A T) \times n(C R))+ \\
& (2+2 \times n(A T))+2 \times(3 \times n(A T)+n(C R)-1)+ \\
& (2 \times n(A T))+1 \\
& =(2 \times n(D M) \times n(A T) \times n(C R))+3+(3 \times n(A T) \times n(C R))+ \\
& (10 \times n(A T))+(2 \times n(C R))
\end{aligned}
$$


Table 5. The general form of CI for TOPSIS (crisp value) for each step

\begin{tabular}{|c|c|}
\hline Step & The general form of CI \\
\hline $\begin{array}{l}\bar{x}_{i j}=\sum_{k=1}^{K} \frac{x_{i j k}}{K} \text { in which } x_{i j k} \text { represents the rating value of the } i \text { th maintenance strategy with the } \\
\text { respective jth factor given by decision-maker } k \text { where } i=1,2,3, \ldots, m, j=1,2,3, \ldots, n \text {, and } k= \\
\qquad 1,2,3, \ldots, K .\end{array}$ & $n(D M) \times n(A T) \times n(C R)$ \\
\hline $\begin{array}{c}\bar{w}_{i j}=\sum_{k=1}^{K} \frac{w_{i j k}}{K} \text { where } \bar{w}_{i} \text { represents the average value of the weight rating corresponding to } \\
\text { the } i \text { th factor from } K \text { decision-makers. }\end{array}$ & $n(D M) \times n(A T) \times n(C R)$ \\
\hline 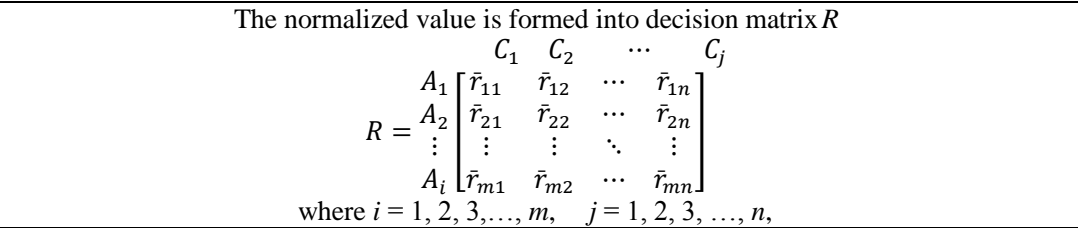 & $2+2 \times n(A T) \times n(C R)$ \\
\hline 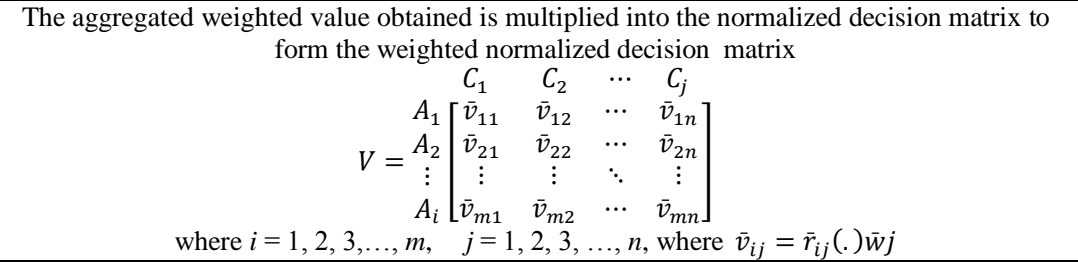 & $n(A T) \times n(C R)$ \\
\hline $\begin{array}{l}\text { According to the TOPSIS algorithm, PIS, } A^{+} \text {and NIS, } A^{-} \text {are identified from the weighted } \\
\text { normalized decision matrix according based on; } \\
\qquad \begin{array}{c}A^{+}=\left\{\bar{v}_{1}^{+}, \bar{v}_{2}^{+}, \cdots, \bar{v}_{n}^{+}\right\} \text {where } \bar{v}_{j}^{+}=\max \left\{\bar{v}_{i j}\right\} \\
\text { where } i=1,2,3, \ldots, m, \quad j=1,2,3, \ldots, n \\
A^{-}=\left\{\bar{v}_{1}^{-}, \bar{v}_{2}^{-}, \cdots, \bar{v}_{n}^{-}\right\} \text {where } \bar{v}_{j}^{+}=\min \left\{\bar{v}_{i j}\right\}\end{array}\end{array}$ & $2+2 \times n(A T)$ \\
\hline $\begin{array}{l}\text { The separation distance of each maintenance strategy from the PIS and NIS is calculated by } \\
\text { using the Euclidean distance as presented; } \\
\qquad \begin{array}{c}d_{i}^{+}=\sum_{j=1}^{n} \sqrt{\left(\bar{v}_{i j}-\bar{v}_{j}^{+}\right)^{2}}, j=1,2, \ldots, n, \\
d_{i}^{-}=\sum_{j=1}^{n} \sqrt{\left(\bar{v}_{i j}-\bar{v}_{j}^{-}\right)^{2}}, j=1,2, \ldots, n,\end{array}\end{array}$ & $2 \times(3 \times n(A T)+n(C R)-1)$ \\
\hline $\begin{array}{l}\text { The measurement of the closeness coefficient is conducted by referring to; } \\
\qquad C_{i}^{*}=\frac{d_{i}^{-}}{d_{i}^{+}+d_{i}^{-}}, 0<C_{i}^{*}<1, i=1,2, \ldots n\end{array}$ & $2 \times n(A T)$ \\
\hline The ranking of the output based on $C_{i}^{*}$ & 1 \\
\hline
\end{tabular}

The general form of CI for [15] of crisp value for each step is as Table 5 .

where $(D M) \in N, n(A T) \in N$ and $n(C R) \in N$.

Based on the simplified general form of CI obtained, the CI for [15] for crisp value when DM is one, alternative and criteria are three $(n(D M)=1, n(A T)=3, n(C R)=3$ ) are;

$$
\begin{aligned}
& \text { CI }_{\text {TOPSIS }, \text { Crisp Value }, n(D M)=1} \\
& =3+(3 \times n(A T) \times n(C R)) \\
& +(10 \times n(A T))+(2 \times n(C R)) \\
= & 3+(3 \times 3 \times 3)+(10 \times 3) \\
= & 66
\end{aligned}
$$

The CI of [15] method for crisp value when DM is three, alternative and criteria are three $(n(D M)=3, n(A T)=$ $3, n(C R)=3$ ) are;

$$
\begin{aligned}
C I_{\text {TOPSIS,Crisp Value }} n(D M)=3 & \\
& =(2 \times n(D M) \times n(A T) \times n(C R))+3 \\
& +(3 \times n(A T) \times n(C R)) \\
& +(10 \times n(A T))+(2 \times n(C R))
\end{aligned}
$$

$$
\begin{aligned}
& =(2 \times 3 \times 3 \times 3)+3+(3 \times 3 \times 3)+(10 \times 3) \\
& \quad+(2 \times 3) \\
& =120
\end{aligned}
$$

Thus, the RCI is obtained by selecting the $\operatorname{Max}(\mathrm{CI})$ first. Then, each of the CI is divided by $\operatorname{Max}(\mathrm{CI})$. The summary of the complexity level for the TOPSIS crisp value of one and three decision-maker is in Table 6.

Table 6. The summary of the complexity level for TOPSIS crisp value of one and three decision-makers

\begin{tabular}{|c|c|c|c|c|}
\hline DM & Big-O & CI & $\operatorname{Max}(\mathrm{CI})$ & RCI \\
\hline Single & $O\left(n^{2}\right)$ & 66 & \multirow{2}{*}{120} & $\frac{66}{120}=0.55$ \\
\cline { 1 - 2 } \cline { 5 - 5 } Group & $O\left(n^{2}\right)$ & 120 & & $\frac{120}{120}=1$ \\
\hline
\end{tabular}

Based on Table 6, Big-O evaluated that [15] method for single and group decision-makers having the same complexity level that is $O\left(n^{2}\right)$. It is known that the same method with a different value of decision-makers will have different complexity levels due to the increasing number of steps involved. Since Big-O evaluated the complexity as the same, RCI is needed to measure precisely the 
complexity level. Based on RCI, a group decision-maker has a higher complexity level compared with a single decision-maker. For this evaluation, it is trivial to know as group decision-makers will have a higher level of complexity compared to a single decision-maker. However, the importance of RCI is seen when evaluating several different methods of decision-making with the same advantage(s) but with different complexity levels where a method with a less complexity level is preferred.

\section{Conclusions}

The importance of proposing a technique to measure the complexity level of the decision-making method is for the decision-maker to choose the best method to be applied. This is because there are several decision-making methods with the same ability. Since complexity may impose many disadvantages such as the time consuming and also the efficiency of the outcome, hence, it is best to use a method with a less complexity level. The initial step of measuring the complexity level of a method is to apply Big-O to the decision-making method. Since based on Big-O there is a lacking (some decision-making methods are ranked equal in terms of complexity level), a relative complexity index (RCI) is proposed. The RCI proposed is based on the primitive operation. After proposed the measuring method, the proposed method is implemented in the decision-making method. As a future recommendation, the implementation of the proposed method is extended to another type of decision-making method such as heterogeneous and multi-granular linguistic term sets.

\section{Acknowledgments}

This research has been supported by the Universiti Teknologi MARA.

\section{REFERENCES}

[1] S. Larsen, M., Manning and P. Pedersen, "Uncovering the hidden costs of offshoring: The interplay of complexity, organizational design, and experience," Strateg. Manag. J., vol. 34, pp. 533-542, 2013.

[2] C. Raaijmakers, A. G. M., Vermeulen, P. A. M., Meeus, M. T. H. and Zietsma, "I need time! Exploring pathways to compliance under institutional complexity," Acad. Manag. J., vol. 58, pp. 85-110, 2015.

[3] F. Marle, L. A. Vidal, and J. C. Bocquet, "Interactions-based risk clustering methodologies and algorithms for complex project management," Int. J. Prod. Econ., vol. 142, no. 2, pp. 225-234, 2013, doi: 10.1016/j.ijpe.2010.11.022.

[4] S. Massanet, J. V. Riera, and J. Torrens, "A new approach to Zadeh's Z-numbers: Mixed-discrete Z-numbers," Inf. Fusion, vol. 53, no. November 2018, pp. 35-42, 2020, doi: 10.1016/j.inffus.2019.06.015

[5] F. Herrera, E. Herrera-viedma, L. Martinez, F. Mata, and J. S. Pedro, "A Multi-Granular Linguistic Decision Model for Evaluating the Quality of Network Services," Intell. Sens. Eval., pp. 71-91, 2004.

[6] F. Herrera, S. Alonso, F. Chiclana, and E. Herrera-Viedma, "Computing with words in decision making: foundations, trends and prospects," Fuzzy Optim. Decis. Mak., vol. 8, no. 4, pp. 337-364, Sep. 2009, doi: 10.1007/s10700-009-90652.

[7] S. Massanet, J. V. Riera, J. Torrens, and E. Herrera-Viedma, "A new linguistic computational model based on discrete fuzzy numbers for computing with words," Inf. Sci. ( Ny)., vol. 258, pp. 277-290, Feb. 2014, doi: 10.1016/j.ins.2013. 06.055 .

[8] M. B. Balasubramanian, N. Lieberman, "Industry learning environments and the heterogeneity of firm performance," Strateg. Manag. J., vol. 412, pp. 390-412, 2010.

[9] G. Sargut and R. McGrath, "Learning to live with complexity," Harv. Bus. Rev., vol. 89, pp. 68-77, 2011.

[10] S. Kanazawa, "A Brief Note on a Further Refinement of the Condorcet Jury Theorem for Heterogeneous Groups," Math. Soc. Sci., vol. 35, no. 1, pp. 69-73, 1998.

[11] Y. P. Jiang, Z. P. Fan, and J. Ma, "A Method for Group Decision Making with Multi-Granularity Linguistic Assessment Information.," Inf. Sci. (Ny)., vol. 178, pp. 1098-1109, 2008.

[12] Drozdek, Data Structure And Algorithms In C++, 2nd Editio. Brooks/Cole (Thomson Learning), 2001.

[13] M. T. Goodrich, R. Tamassia, and D. M. Mount, Data Structures \& Algorithms in $C++, 2$ nd editio. Hohn Wiley \& Sons, Inc, 2011.

[14] H. S. Shih, H. J. Shyur, and E. S. Lee, "An extension of TOPSIS for group decision making," Math. Comput. Model., vol. 45, no. 7-8, pp. 801-813, 2007, doi: 10.1016/j.mcm.2006.03.023.

[15] S.-H. Ding and S. Kamaruddin, "Assessment of distance-based multi-attribute group decision-making methods from a maintenance strategy perspective," J. Ind. Eng. Int., 2014, doi: 10.1007/s40092-014-0078-2. 